\title{
PREPARATION AND CHARACTERIZATION OF MAGNESIUM/ALUMINIUM LAYERED DOUBLE HYDROXIDE AS FILLER IN LOW-DENSITY POLYETHYLENE COMPOSITE
}

\author{
NUR ATHIRAH ZULKIFLI, MOHD AIDIL ADHHA ABDULLAH*, AND MAZIDAH MAMAT
}

Faculty of Science and Marine Environment, Universiti Malaysia Terengganu, 21030 Kuala Nerus, Terengganu, Malaysia.

* Corresponding author: aidil@umt.edu.my

http://doi.org/10.46754/umtjur:2021.01.001

\begin{abstract}
Polymer had been widely used in industries nowadays. However, the properties of the polymer itself are limited to a particular application. This study describes synthetic clay, layered double hydroxide (LDH), as a filler in low-density polyethylene (LDPE) composite. LDHs of magnesium/ aluminium-dodecyl sulfate (Mg/Al-DS) and its grafted with triethoxymethylsilane (TEMS), (TEMSg-Mg/Al-DS) were synthesized through co-precipitation and salinization reaction methods. The presence of alkyl group, $\mathrm{v}(\mathrm{C}-\mathrm{H})$ in both $\mathrm{LDH}$ had confirmed through Fourier transform infrared (FTIR). The appearance of peaks in FTIR spectra within the absorbance range of $2800-2930 \mathrm{~cm}^{-1}$ indicates a successful surface modification of LDH, supported by the changes of interlayer spacing and the presence of carbon from X-ray diffractogram and CHNS elemental analysis, respectively. The synthesized LDH was mixed with LDPE via melt intercalation method. The LDH modification resulted in higher interaction and compatibility between the LDPE matrix and LDH by the formation exfoliated type of nanocomposites, as suggested by XRD analysis.
\end{abstract}

Keywords: Layered double hydroxide, co-precipitation method, triethoxymethylsilane; nanocomposites, salinization.

\section{Introduction}

Polymers are broadly used materials that can be found most in our daily life, and it is essential because of their various applications in science, technology, and industries (Namazi, 2017). A specific polymer property for new applications is more easily produced by compounding commercially available polymer rather than synthesizing a new polymer. A study on reinforcing filler such as clay has attracted scientists worldwide to tailor the properties of polymer for a specific application.

Layered double hydroxide (LDH) is a synthetic clay that can be an alternative to natural clay such as montmorillonite (MMT) as filler in polymer nanocomposites. MMT, which is known as 2:1 types of clay minerals commonly utilized by polymer technologists to improve the properties of a polymer. However, MMT's chemical compositions differ depending on the location it is obtained, which may affect the final properties of polymer composites. LDH advantages such as manageable chemical composition and charged layers and ion exchange capacity can improve with surfactants to be compatibilized with organic polymer (Eili et al., 2012). With latest understanding of the fundamental about $\mathrm{LDH}$, this layered material, which commonly consists of approximately 10um-size multilayer stack (Gianellis, 1996), can be separated into individual nano-layer, hence increasing the surface area for interaction with polymer matrixes. The introduction of nanoparticle in polymer composites increased in terms of optical (Loste et al., 2019), thermal (Kurt and Koca, 2015), mechanical (Bek \& Jeon, 2010) and permeability (Ramesh and Punithamoorthy, 2019) properties that must give a variety of applications in many fields.

However, LDH is hydrophilic and not compatible with an organic polymer resulted in the phase-separated and agglomeration of clay platelets or known as conventional polymer-clay composites. By introducing a functionalized organic surface modification via physical and chemical reaction onto $\mathrm{LDH}$, the compatibility between the polymer and LDH can be improved 
(Zhang et al., 2016). The compatibility between polymer and LDH resulted in LDHfunctionalized layer stacks separated and distributed into individual layer throughout the organic polymer matrix.

Previous literature shows that a small organoclay loading $(1-5 \% \mathrm{wt})$ in polymers provides substantial benefits in composites' properties. The tensile, flexural, and thermal properties are improved by $30-40 \%$ with the presenceoforganoclayin the compositecompared to the pristine polymer (Shunmugasamy et al., 2015). However, at higher organoclay loading can lead to a reversal of the trends and reduce the mechanical properties of nanocomposites due to entrapment of air porosity and agglomeration of the clay (Bee et al., 2017).

In this work, we study the compatibility of $\mathrm{Mg} / \mathrm{Al}$ layered double hydroxide with LDPE by benchmarking the types of composites obtained when introducing LDH into the LDPE matrix.

\section{Materials and Methods}

\section{Materials}

Metal salts, magnesium nitrate hexahydrate, $\mathrm{Mg}\left(\mathrm{NO}_{3}\right)_{2} \cdot 6 \mathrm{H}_{2} \mathrm{O}$ and aluminium nitrate nonahydrate $\mathrm{Al}\left(\mathrm{NO}_{3}\right)_{3} \cdot 9 \mathrm{H}_{2} \mathrm{O}$, and chemicals, sodium dodecyl sulfate (SDS), low-density polyethylene, and sodium hydroxide purchased from Sigma-Aldrich (M) Sdn. Bhd. Moreover, distilled water utilized throughout the experiments. All LDHs prepared via a coprecipitation method.

\section{Synthesis of Mg/Al-Dodecylsulphate LDH}

Mother liquor solution was prepared by mixing, magnesium nitrate hexahydrate and aluminium nitrate nonahydrate with a metal molar ratio of 3:1 in $90 \mathrm{~mL}$ distilled water. $50 \mathrm{~mL} 0.50 \mathrm{M}$ sodium dodecyl sulphate solution then added to the mother liquor solution. 2.0M sodium hydroxide solution added dropwise until $\mathrm{pH} 10$ obtained. The mixture was aged at $80^{\circ} \mathrm{C}$ in an oil bath for 12 hours and afterwards, the resultant slurry was filtered, washed with distilled water, and dried in an oven. This mono functionalized LDH was labelled as Mg/Al-DS LDH.

\section{Grafting triethoxymethylsilane (TEMS) onto $\mathrm{Mg} / \mathrm{Al}-\mathrm{DS} \mathrm{LDH}$}

Salinization of LDH conducted using TEMS via a sialylation reaction with the hydroxyl group in LDH. A similar procedure was adopted to synthesize $\mathrm{Mg} / \mathrm{Al}-\mathrm{DS} \mathrm{LDH}$ with the addition of $50 \mathrm{~mL}$ TEMS solution into the mother liquor before titration with sodium hydroxide. This twice-functionalized LDH was labelled as TEMS-g-Mg/Al-DS LDH.

\section{Preparation of LDPE/LDH nanocomposites}

Low-density polyethylene (LDPE) composites with various LDH loads (1-5wt\%) were prepared via melt intercalation using a Haake internal mixer, at $180^{\circ} \mathrm{C}$, screw speed of $60 \mathrm{rpm}$ with residence time of 10 minutes.

\section{Materials Characterization}

The orientation of the LDH layer in composites based on diffraction of d003 plane using X-ray diffractometer, Rigaku Mini Flex II using $\mathrm{CuKa}$ radiation at wavelength 0.154 $\mathrm{nm}$. The scanning rate used in this study was $2^{\circ} \mathrm{min}^{-1}$. The interactions of the clay minerals with inorganic or organic compounds were determined using Perkin Elmer spectrum 100 within the wavelength range $400-4000 \mathrm{~cm}^{-1}$. The sample was prepared using $\mathrm{KBr}$ pressed pellet technique. LECO CHNS-932 Elemental analyser provides the quantitative analysis of carbon presence in LDH. Composite specimens for XRD analysis were prepared using Haake Minijet injection moulding.

\section{Results and Discussion}

\section{FTIR Analysis}

Figure 1 shows FTIR spectra of synthesized LDHs. The LDHs displayed similar features due to water and hydroxyl vibration modes from 4000 to $1600 \mathrm{~cm}^{-1}$. The hydroxyl stretching 
gives strong and broad absorption bands at 3437 and $3458 \mathrm{~cm}^{-1}$ and deformation vibration of the interlayer water at 1637 and $1639 \mathrm{~cm}^{-1}$ for $\mathrm{Mg} / \mathrm{Al}-$ DS and TEMS-g-Mg/Al-DS LDH, respectively. The broadness of the hydroxyl stretch region attributed to the presence of hydrogen-bonded hydroxyl groups. For TEMS-g-Mg/Al-DS LDH, the broad peak of $\mathrm{OH}$ is shifted from 3458 to lower wavenumber which is 3437 compared to $\mathrm{Mg} / \mathrm{Al}$-DS $\mathrm{LDH}$ which means that grafted LDH with TEMS and the modification with SDS give significant influence to the shifting of wavenumber (Tao et al., 2009).

The presence of the alkyl group from DS structure represent by the $\mathrm{C}-\mathrm{H}$ aliphatic stretching vibration implied by the observation of the triplet band at 2800-3000 $\mathrm{cm}^{-1}$. Weak peaks at 1467 and 1468 for the bending mode of $\mathrm{CH}_{2}$ group of DS. S-O and $\mathrm{S}=\mathrm{O}$ stretching modes of dodecyl sulphate at 1216 and 1217 . The absorption peak at $1383 \mathrm{~cm}^{-1}$ indicated the presence of nitrate anion in the interlayer gallery of LDH, which co-existed with DS anion.

The grafted TEMS onto LDH brings a vibration of Si-O-M (M $=\mathrm{Mg} \& \mathrm{Al})$ at $1016 \mathrm{~cm}$ which appeared in TEMS-g-Mg/Al-DS LDH spectrum. This finding agrees with XRD results, and elemental analysis supported the successful TEMS grafted onto LDH layers.

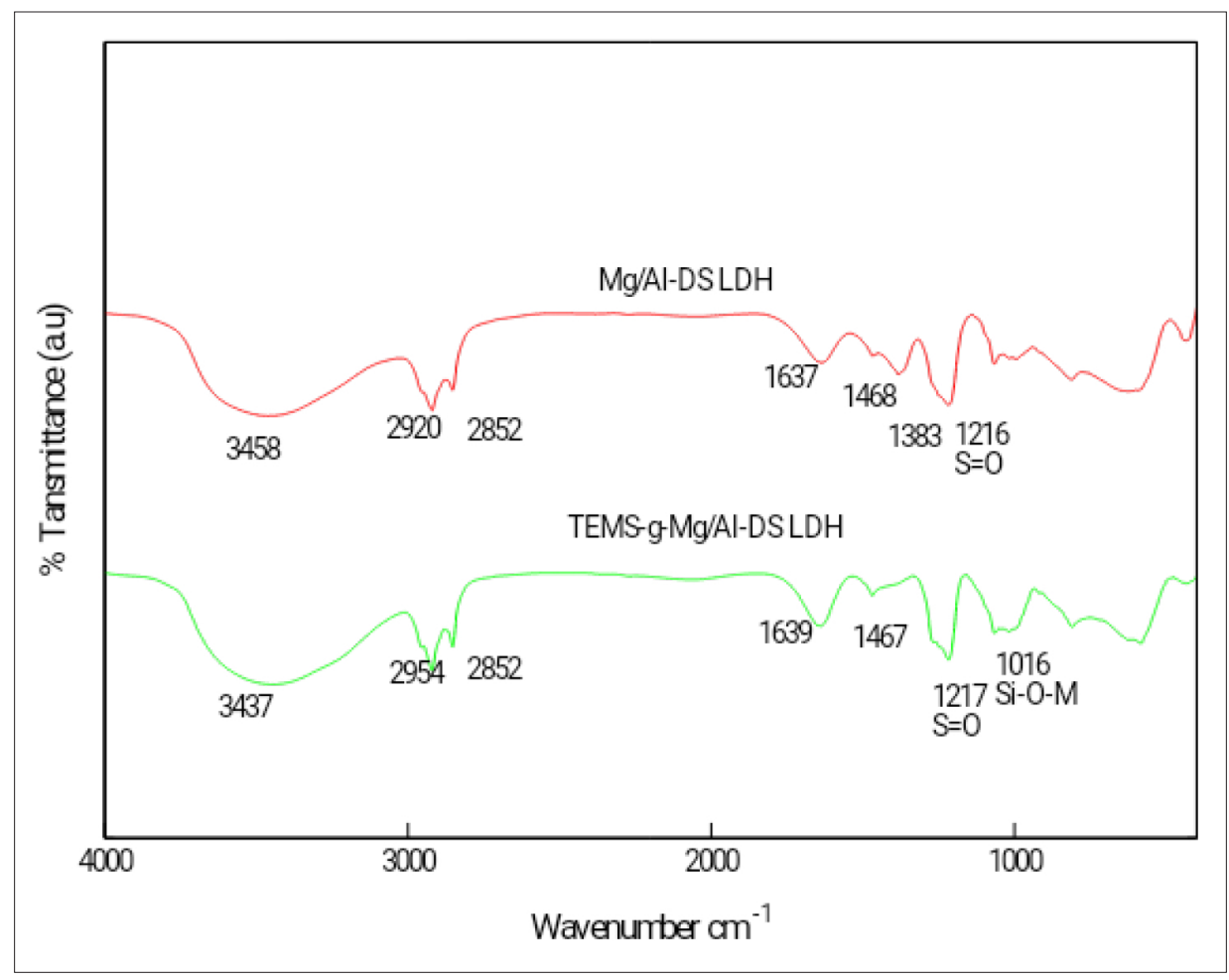

Figure 1: FTIR spectra of LDHs

\section{Elemental Analyses}

Based on the percentage of carbon, there are $229 \mathrm{mmol} \mathrm{DS}$ anion presence in $100 \mathrm{~g} \mathrm{Mg} / \mathrm{Al}-$ DS LDH. However, the presence of DS content decreases from $33 \%$ to $32 \%$ after the grafting of
TEMS onto LDH suggested the grafting TEMS altered the charge of the LDH. The presence of $1 \%$ nitrogen in $\mathrm{Mg} / \mathrm{Al}-\mathrm{DS} \mathrm{LDH}$ and $4 \%$ in TEMS-g-Mg/Al-DS LDH suggested the nitrate anion co-exist as the interlayer anion in LDHs as supported by FTIR. 


\section{PXRD Results}

From X-ray diffractograms of LDHs (Figure 2), the $d_{003}$ diffraction plane peaks of $\mathrm{Mg} / \mathrm{Al}$ DS LDH and TEMS-g-Mg/Al-DS LDH, were
2.63 and $3.24 \mathrm{~nm}$, respectively. The higher value of interlayer spacing for TEMS-g-Mg/Al-DS LDH possibly due to the grafting of TEMS onto interlayer of LDH (Bee et al., 2017).

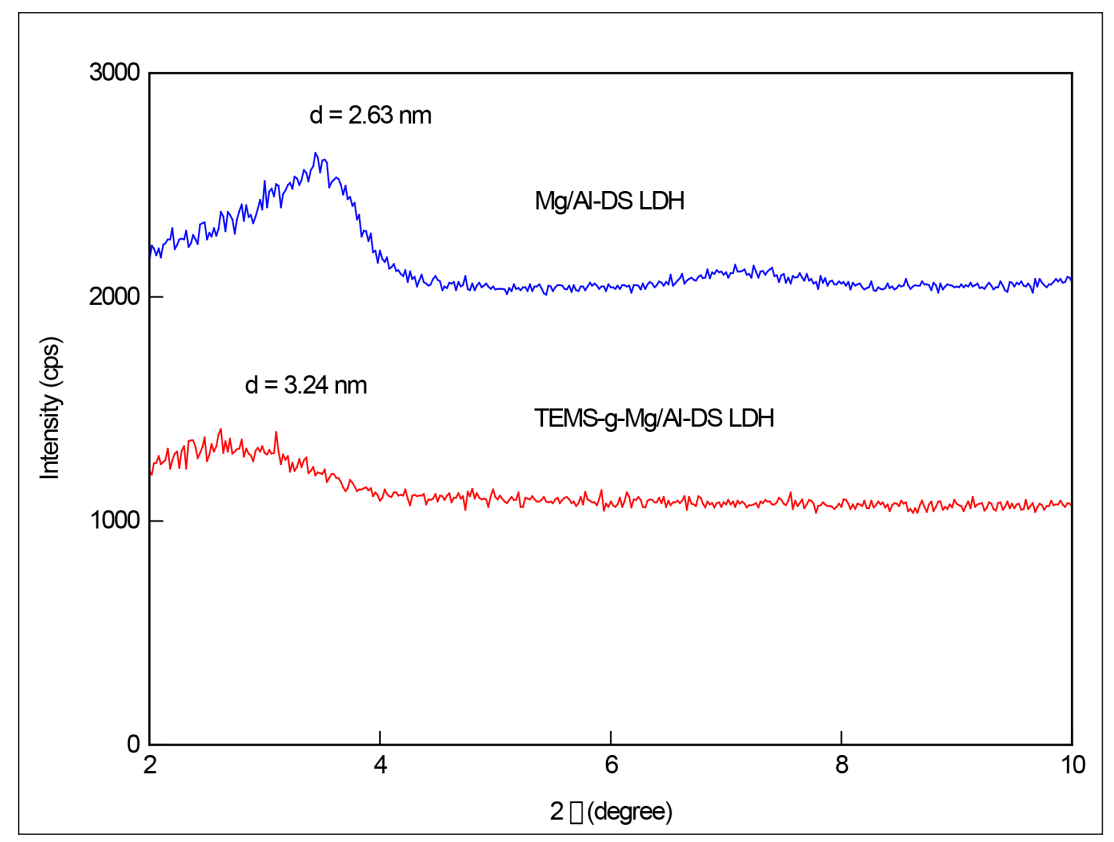

Figure 2: X-Ray diffractograms of LDHs

\section{Surface morphology of LDPE composites}

After the LDHs were melt blended with LDPE, the dispersion level of $\mathrm{LDH}$ layer into the LDPE matrix were studied. The basal spacing from the diffraction $d_{003}$ plane of $L D H$ was used for comparison. The surface interaction is the primary factor affecting the composite properties. Maximum degree of dispersion and interaction occurred when LDH layers exfoliated or delaminated in the polymer matrix. Intercalated types of nanocomposite have less surface interaction with the platelet structure remains with a higher interlayer spacing due to the insertion of polymer in the interlayer of LDH (Suresh et al., 2016). The conventional composite is considered to have the lowest interaction due to only the outer layered surface of LDH interacting with polymer matrices. 


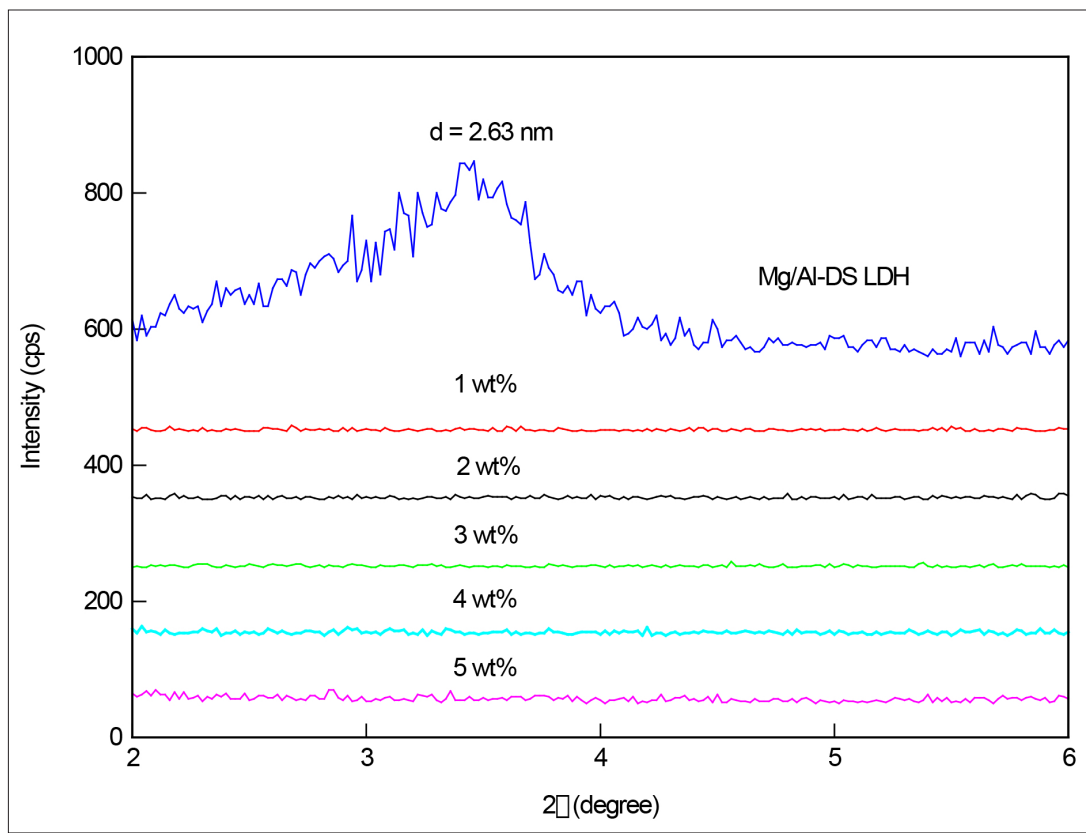

Figure 3: X-ray diffractograms of $\mathrm{Mg} / \mathrm{Al}-\mathrm{DS} \mathrm{LDH}$ and its composites at a different weight percent.

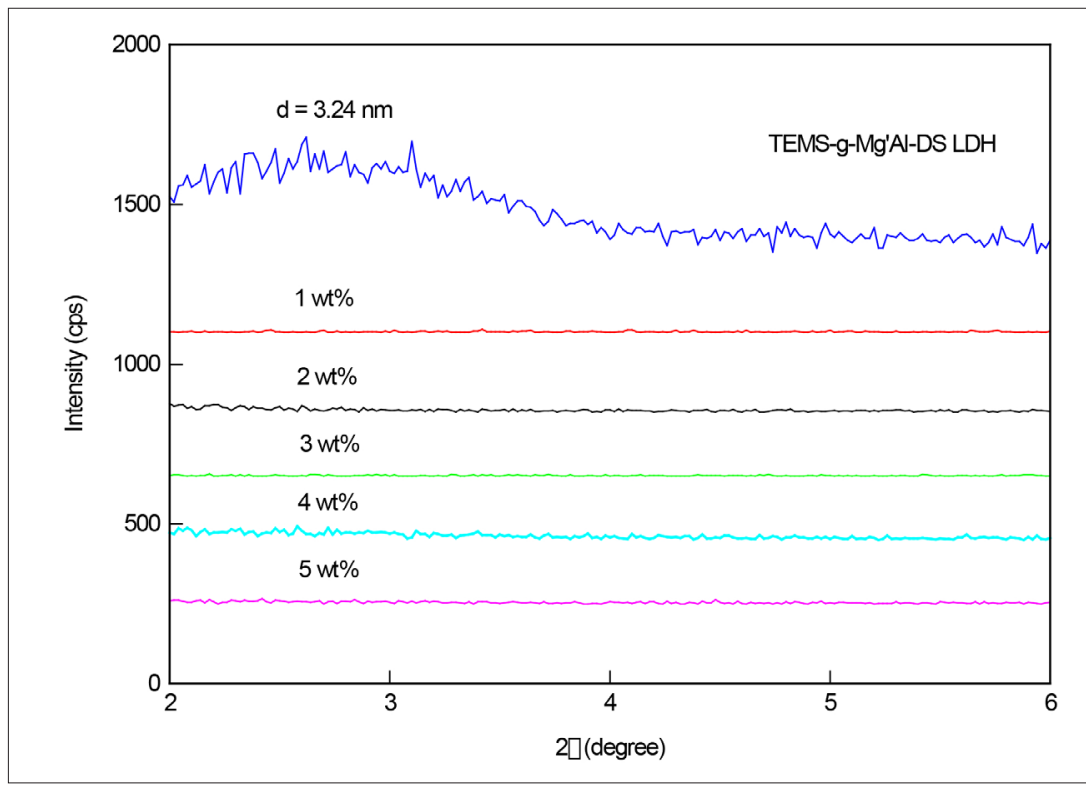

Figure 4: X-ray diffractograms of TEMS-g-Mg/Al-DS LDH and its composites at a different weight percent.

Figures 3 and 4 show the X-ray diffractograms of composites after LDHs mixed with LDPE. The diffraction $d_{003}$ plane of the LDH layer for both composites disappeared, indicated the highest degree of layered hydroxide interaction with LDPE, which caused the loose interaction among layered hydroxide layers due to the presence of LDPE matrix between the LDH layers. The presence of organic surfactant (dodecyl sulphate) in both LDH had increased 
its hydrophobicity. The less abundant hydroxyl groups on the TEMS-g-Mg/Al-DS surfaces after the grafting reaction took place improves the compatibility with LDPE. As there was no $d_{003}$ diffraction plane indicate the layers of LDH are not in a structured stack for $1-5 \mathrm{wt}$. $\%$, this suggested the formation of exfoliated composites (Suresh et al., 2016).

\section{Conclusion}

Organo layered double hydroxide of $\mathrm{Mg} / \mathrm{Al}-\mathrm{DS}$ system was successfully synthesized and grafted with TEMS. Mono and twice- functionalized LDHs have potential as reinforcing filler for LDPE by forming composites with the highest degree of interaction between galleries of LDH and intercalation of LDPE into it. Exfoliated nanocomposites expected to have an improvement in properties.

\section{Acknowledgements}

The authors would like to express gratitude to Universiti Malaysia Terengganu for providing research facilities for this research project.

\section{References}

Bee, S., M.A.A. Abdullah., Mazidah Mamat., Bee, S., Sin, L. T., Hui, D., \& Rahmat, A. R. (2017). Characterization of silylated modified clay nanoparticles and its Functionality in PMMA. Composites Part $B$ : Engineering, 110, 83-95.

Eili, M., Shameli, K., Ibrahim, N. A., \& Yunus, W. M. (2012). Degradability Enhancement of Poly(Lactic Acid) by stearate-Zn3Al LDH nanolayers. International Journal of Molecular Sciences, 13(7), 7938-7951.

Giannelis, E. P. (1996). Polymer layered silicate nanocomposites. Advance Materials, 8, 29-35.

Jeon, I., \& Baek, J. (2010). Nanocomposites derived from polymers and inorganic nanoparticles. Materials, 3(6), 3654-3674.
Kurt, A. \& Koca, M. (2015). Optical Properties of Poly(2-(5-bromo benzofuran-2-yl)2-oxoethyl methacrylate)/Organoclay Nanocomposites. Arabian Journal for Science and Engineering, 40, 2975-2984.

Loste, J., Lopez-Cuesta, J-M., Billon, L., Garay, H. and Save, M. (2019). Transparent polymer nanocomposites: An overview on their synthesis and advanced properties. Progress in Polymer Science, 89, 133-158.

Namazi, H. (2017). Polymers in Our Daily Life. Journal of BioImpacts, 7(2), 73-74.

Ramesh, S. \& Punithamoorthy, K. (2019). Synthesis, characterization and gas permeability properties of a novel nanocomposite based on poly(ethyleneco-vinyl acetate)/polyurethane acrylate/ clay. Journal of Materials Research and Technology, 8 (5), 4173-4181.

Shunmugasamy V., Xiang C., Gupta N. (2015) Clay/Polymer Nanocomposites: Processing, Properties, and Applications. In: Kim CS., Randow C., Sano T. (eds) Hybrid and Hierarchical Composite Materials. Springer, Cham

Suresh, K., Kumar, R. V., \& Pugazhenthi, G. (2016). Processing and characterization of polystyrene nanocomposites based on CoAl layered double hydroxide. Journal of Science: Advanced Materials and Devices, 1(3), 351-361.

Tao, Q., He, H., Frost, R. L., Yuan, P., \& Zhu, J. (2009). Nanomaterials based upon silylated layered double hydroxides. Applied Surface Science, 255(7), 4334-4340.

Zhang, H., Zhang, J., Yun, R., Jiang, Z., Liu, H., \& Yan, D. (2016). Nanohybrids of organomodified layered double hydroxides and polyurethanes with enhanced mechanical, damping and UV absorption properties. RSC Advances, 6(41), 34288-34296. 